\title{
Consultation on basic asthma research strategy
}

\author{
T.H. Lee
}

There have been major advances in the understanding of the mechanisms and genetics of asthma in recent years. Some findings have already led to the development of new therapeutic agents, and others may be the basis for further new drug entities. However, while much has been achieved, many challenges remain. Prevalence of the condition continues to grow, and in the UK, an estimated 8 million people are diagnosed as having asthma, with 5 million people receiving treatment. The cost of asthma to the National Health Service in the UK is $>£ 850$ million ( $€ 1,360$ million) a year [1].

In the light of the recent rapid expansion of knowledge, the National Asthma Campaign in the UK decided to gain a consensus on the priorities for asthma research over the next 5 yrs. The executive summary was published in Clinical Experimental Allergy [2] and the full document [3], which encompasses consensus views on current research and recommendations for future work, is available from the Research Director, National Asthma Campaign, Providence House, Providence Place, London N1 0NT, UK.

An executive group of asthma scientists and representatives from the major funding agencies was convened. A representative from the Netherlands Asthma Foundation (Leusden, the Netherlands) provided an international perspective. The executive group identified seven major areas in basic asthma research that need detailed consideration by working groups. These were: genetics; early life events; environmental impact; immunology and immunotherapy; inflammation and anti-inflammation; airway remodelling; and interface between academia and industry. Each working group comprised outstanding asthma and nonasthma scientists. They were advised that their recommendations should not be constrained by financial considerations, but should be driven by science and vision.

The following generic key recommendations were made: greater attention should be given to the different phenotypes of the disease; greater emphasis should be put on longitudinal studies; existing hypotheses on the causation of asthma should be tested to provide clarity; research should be considered in the context of a continuum from bench to patient; collections of deoxyribonucleic acid (DNA), ribonucleic acid and lung tissue should be coordinated centrally

Correspondence: T.H. Lee, Dept of Allergy and Respiratory Medicine, Guy's Hospital, London, SE1 9RT, UK. Fax: 44 2074038640. E-mail: tak.lee@kcl.ac.uk to maximise research opportunities; cooperative multidisciplinary networks should be established, although methodologies will have to be standardised; greater use should be made of existing databases, which should be collated across centres both nationally and internationally; and partnerships between academia and industry should be encouraged.

The exploration of opportunities for collaborative funding of major research projects between Government, research councils, charities (including joint funding with related charities in Europe) and industry was considered important. It was considered imperative for large-scale, lengthy, multicentre longitudinal studies to have clear objectives, robust design, transparent milestones and a well-defined exit point for conclusion. To ensure that a steady supply of asthma researchers progress through the career structure and to provide greater job security, it was suggested that more long-tenure posts should be established for clinical and nonclinical scientists and training fellows.

Establishing whether allergy and asthma can be prevented in infancy (primary prevention) was considered a high priority. It was also considered important to understand the mechanisms for remission of asthma in adolescence. Several questions were raised in relation to the perpetuation of asthma, and included how and whether inflammation and immunology contribute to chronicity of disease, what is the precise role of airway remodelling and whether it is reversible or progressive. Occupational asthma was proposed as a useful model to investigate mechanisms of initiation, perpetuation and resolution of the condition.

Asthma genetics: Combining genomic and proteomic approaches might provide a way forward. The likely advances in the post-genome era need urgent attention.

Early life events and the development of asthma: A range of agents could be examined, including allergens, microbials and nutrients/diet. Studies require very careful design. Intervention studies on allergen exposure in early life are already underway and it was recommended that the results from these studies should be evaluated before more resources are committed to this specific question. Occupational asthma might represent an important model for the investigation of asthma onset, particularly in older people, and also for mechanisms of resolution.

Inflammation and anti-inflammation in asthma: More research needs to be conducted in people with more symptomatic asthma and the mechanism of chronicity should be investigated. With regard to tissue sampling in patients with severe disease, in view 
of ethical considerations, development of less invasive investigative techniques is a priority. Translational research using novel therapeutic agents to test specific hypotheses should be encouraged. Defects in mechanisms of resolution in inflammation could be a key factor in the chronicity of asthma. The manner in which the inflammatory processes of asthma interact with environmental influences remains unknown. Animal models are useful in inflammation research, but are not helping clarify any aspects of the chronicity of the process. More representative models need to be developed.

Immunology and immunotherapy in asthma: Future work is needed to help understand the regulation of inflammatory and anti-inflammatory T-cells, including antigen-presenting cell-derived signals upon which T-cell activation is critically dependent. The role of T-cells, if any, in the chronicity of asthma needs to be assessed. The recent development of humanised nonanaphylactic monoclonal immunoglobulin (Ig)E antibodies will allow a logical assessment of the role of $\mathrm{IgE}$ in asthma, especially in more severe disease. The hypothesis that specific immunotherapy (SIT) might have disease-modifying effects requires detailed re-appraisal and there is a need to compare the influence of SIT on the long-term evolution of established asthma in adults with that of drug therapy. Novel forms of immunomodulation require testing, including: bacterial type DNA that is intended to switch on T-helper (Th)1 and suppress Th2 responses; agents directed against individual cytokines; and the use of probiotic bacteria designed to modify the intestinal microflora.

Airway remodelling in asthma: Larger studies of airway anatomy and function are required to provide a clear link between airway remodelling and physiological changes, including studies in children. Matrix biology is clearly an important area of knowledge that could be developed and validation of noninvasive markers of matrix turnover are especially important. Noninvasive methods of analysing morphological changes in the airway are already used in the study of chronic obstructive pulmonary disease, including emphysema, and are likely to become increasingly important in the study of asthma.

Interactions between academia and industry in asthma research: Academic institutions should continue to perform the hypothesis-led research that has proved so successful in elucidating new targets in the treatment of disease. Academic centres can provide help with identifying surrogate markers for disease, patient stratification and phenotyping, imaging technology, proof of concept studies, target validation and disease monitoring. Some state-ofthe-art technology, such as genomic screens and proteomics, are too expensive for academic centres, making links with industrial partners a critical element of research into asthma genetics. Interactions between research groups, clinicians and industry can help to establish dialogue on clinical needs, identify key discovery targets and debate drug development strategies. One important area for discussion is how to define clinical outcome measures for new disease-modifying drugs that will be predictive of the long-term outcome of the disease. Many large pharmaceutical trials have investigated single interventions in the management of young children with wheezing illnesses. The pharmaceutical industry could be encouraged to support longer-term follow-up of such patients beyond the initial study period. Followup covering the watershed area from 10-15 yrs, where there is a gap in observational cohort studies at a time when asthma remission is moderately frequent, would be of particular value.

The consultation achieved its aim of providing novel and reasoned insights into the way forward for basic asthma research in the short and medium term, for the benefit of people with asthma. Although the National Asthma Campaign sought information to help with its own decisions on research funding, the consultation has a wider relevance to all those invloved in funding and carrying out research in the asthma field.

\section{References}

1. National Asthma Campaign. Out in the open: a true picture of asthma in the UK today. Asthma $J$ 2001; 6: Suppl.

2. Lee T, Barnes J. Where next in basic asthma research? Clin Exp Allergy 2002; 32: 499-506.

3. National Asthma Campaign 2002. Where next in basic asthma research? London, UK, Asthma Enterprises Ltd, 2002. 